\title{
The transfer RIB lines to the DESIR facility at GANIL-SPIRAL2
}

\author{
Luc Perrot $^{1 \mathrm{a}}$, Hamda Cherif ${ }^{1}$, on behalf of the DESIR collaboration \\ ${ }^{1}$ CNRS-IN2P3, Université Paris Sud, IPNO, 91406 Orsay, France
}

\begin{abstract}
The new ISOL facility SPIRAL2 is currently being built at GANIL, Caen France. SPIRAL2 will produce a large number of new radioactive ion beams (RIB) at high intensities. The DESIR facility will receive beams from the upgraded SPIRAL1 facility of GANIL (stable beam and target fragmentation), from the S3 Low Energy Branch (fusion-evaporation and deep-inelastic reactions) and from the SPIRAL2 production cave (n-induced fission of ${ }^{238} \mathrm{U}$, nucleon transfer and fusion-evaporation reactions). In order to deliver the RIB to the experimental set-ups installed in the DESIR hall, 110 meters of beam line have to be designed, originating from 3 different facilities. This paper will focus on the studies which have been done on these transfer lines: beam optics and errors calculations, quadrupoles, diagnostics and mechanical designs.
\end{abstract}

\section{The DESIR facility within the SPIRAL2 project}

SPIRAL2 is a major extension project of the GANIL facility in Caen, France dedicated to the production of heavy ion beams at $\mathrm{mA}$ intensities and of very exotic nuclides. With SPIRAL2, the French and International communities will make decisive steps in the understanding of the atomic nucleus and of the nucleosynthesis processes occurring in astrophysics. The collection of nuclear data will help preparing the next generation of nuclear reactors and the production of new isotopes suitable to nuclear medicine will be investigated. In addition, a high intensity fast neutron source will open new research domains in material science [1]. The SPIRAL2 facility will produce a large number of new radioactive ion beams (RIB) at high intensity. These beams will be produced using a new linear accelerator that will deliver deuterons up to $40 \mathrm{MeV}$ at $5 \mathrm{~mA}$ intensity, protons up to $33 \mathrm{MeV}$ at $5 \mathrm{~mA}$ and ions with $\mathrm{A} / \mathrm{Q}=3$ up to $14.5 \mathrm{MeV} / \mathrm{u}$ at $1 \mathrm{~mA}$ (see fig. 1) [2].

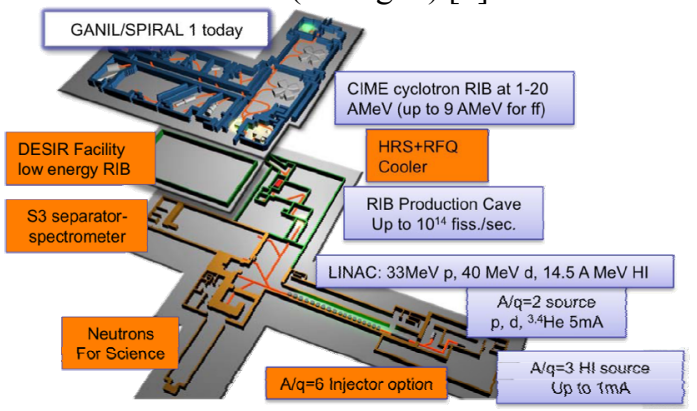

Figure 1. Scheme of the SPIRAL2 facility.

\footnotetext{
${ }^{a}$ Corresponding author: perrot@ipno.in2p3.fr
} 
The DESIR (Désintégration, Excitation et Stockage des Ions Radioactifs = Decay, Excitation and Storage of Radioactive Ions) facility will receive beams from the SPIRAL2 production cave where fission of ${ }^{238} \mathrm{U}$ nuclei, fusion-evaporation, nucleon transfer as well as deep-inelastic reactions will take place [3]. Additionally, RIB from the S3 separator spectrometer of SPIRAL2 will be delivered to DESIR through its low energy branch (S3-LEB). They will be produced in fusion-evaporation, transfer and deep-inelastic reactions, and will notably consist in refractory elements. Finally RIB produced in the fragmentation of high-intensity heavy ions and/or thick targets at the upgraded SPIRAL1 facility will also be available at DESIR [4].

Nuclear physics as well as fundamental weak-interaction physics and astrophysics questions will be addressed by means of laser spectroscopy, decay studies, mass spectrometry and complementary trap-assisted measurements. Experience at other ISOL facilities evidences that ion beams with a high degree of purity are required to push experiments towards the limits of nuclear stability $[5,6,7]$.

\section{Beam dynamics along the DESIR transfer lines}

Coming from the 3 various injection locations (the SPIRAL2 production cave, S3-LEB and SPIRAL1), 110 meters of beam line have to be designed in order to deliver the RIB to the experimental set-ups installed in the DESIR hall. These interconnected lines must be safe, robust, cheap, and their tuning easy and reproducible.

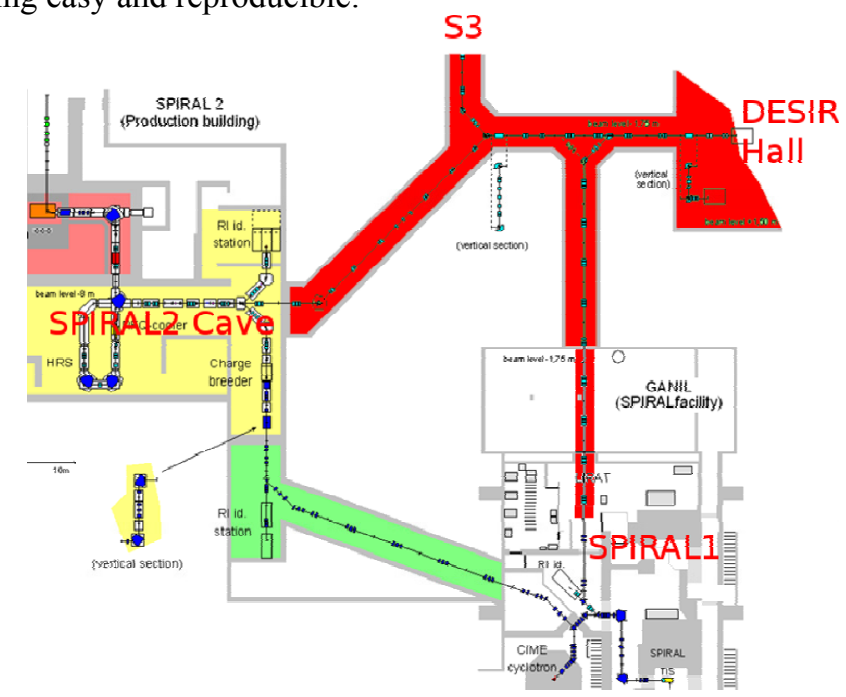

Figure 2. Scheme of the transfer beam line from SPIRAL2 production cave, S3-LEB and SPIRAL1 up to DESIR experimental hall (red background area). Beam line lengths: SPIRAL2 cave to DESIR hall $=54 \mathrm{~m}, \mathrm{~S} 3$ to interconnection $=11.3$, SPIRAL1 to interconnection $=34.7 \mathrm{~m} .10$ meter inside the experimental hall .

The maximum beam energy will be $60 \mathrm{keV}$ and its transverse maximum geometric emittance will be $80 \pi . m m . m r a d$. Only singly-charged ions will be transported, for which electrostatic optical devices are better suited [8,9]. Careful studies of the beam dynamics were performed using the CEA TraceWin code [10]. This code allows to run large and close to real condition simulations, to produce field maps of optical elements, to perform diagnostics location optimizations, errors studies, corrections and feed-backs [11]. Quadrupoles and deflectors alignments have to be better than $0.1 \mathrm{~mm}$, field stability precision better to $1 \%$, rotation better to $\pm 0.1^{\circ}$. Diagnostics position must be better than $\pm 0.5 \mathrm{~mm}$ and beam profiles precision must be lower than $\pm 0.5 \mathrm{~mm}$. Using these conditions, the beam stability in the line is estimate to be less than $1 \mathrm{~mm}$ (orbit and size). It is a compromise between engineering costs, beam characteristics and losses.

Figure 3 shows the particle power density in the horizontal plane along the transfer line linking the SPIRAL2 production cave to the DESIR experimental hall. 


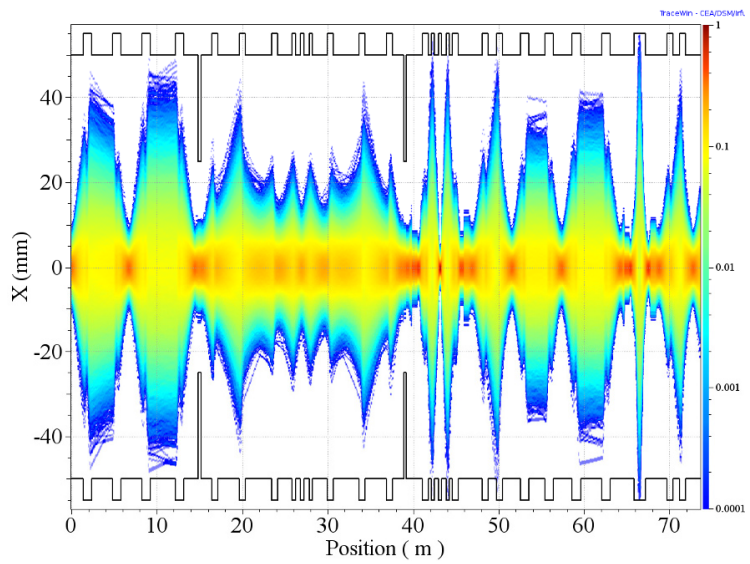

Figure 3. Particle power density in the horizontal plane along the transfer line from the beam matching point in the SPIRAL2 production cave up to the DESIR experimental hall.

Making use of the knowledge and long experience on electrostatic quadrupoles [12, 13], we have studied and designed a quadrupole optimized with respect to the maximum beam emittance and energy. Various electrode shaping (circular and hyperbolic) or field cut-off rings (aperture and distances with the electrode) have been studied using the Opera-3D simulation tool [14]. Then, calculated field map were used and compared directly in the TraceWin simulations of the entire transfer beam line. We will use quadrupoles with aperture to $110 \mathrm{~mm}$, length to $175 \mathrm{~mm}$ with a circular shaping (low cost compare to hyperbolic shape). Rings will be $2 \mathrm{~mm}$ thick, $110 \mathrm{~mm}$ in aperture located at $27.5 \mathrm{~mm}$ to the electrode.

Finally, the precise design of the electrostatic devices of the transfer beam line was used in a full mechanical integration. A quadrupoles triplet with X-Y steerers is composed by 2 vacuum chambers (see figure 4). The first one contains 2 quadrupoles; the second contains one quadrupole with X-Y steerers. These designs are applied along the beams lines in order to form sets of quadrupoles doublets or singles with steerers.

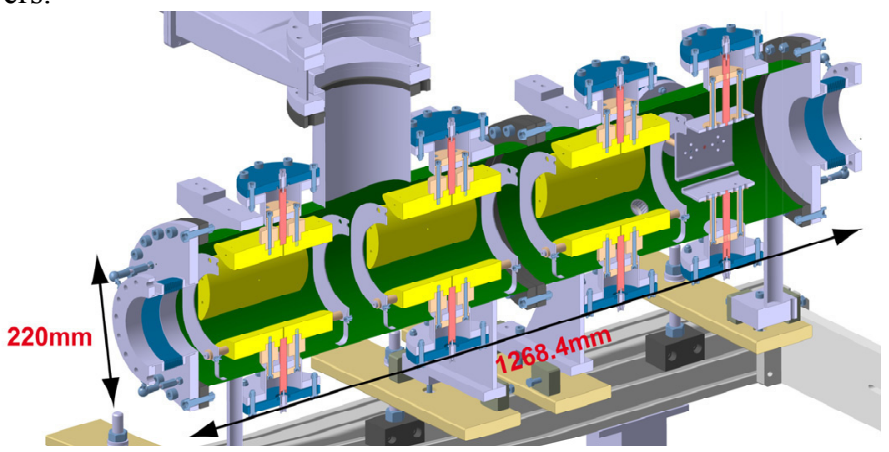

Figure 4. Mechanical design of a standard optical ensemble for the DESIR transfer beam lines with three electrostatic quadrupoles and one X-Y steerers. Length of the vacuum chamber is $1086.4 \mathrm{~mm}$ and internal diameter is $220 \mathrm{~mm}$ (green part). Full length is $1268.4 \mathrm{~mm}$.

A prototype of such a quadrupole triplet with a set of X-Y steerers is now under construction at IPN Orsay. It will be coupled in march 2014 at the CEN Bordeaux-Gradignan laboratory to a radiofrequency quadrupole cooler and buncher to be installed at the DESIR facility. These tests will give us some clear answers about costs, manufacturing process, electric and vacuum characteristics. They will finally allow us to validate the beam dynamics characteristics (tuning, high order contributions). 


\section{Conclusion}

We have presented here a short introduction of the SPIRAL2 and DESIR project. DESIR will be a new and extended experimental area dedicated to the study of the fundamental properties of new exotic radioactive nuclides to be produced at SPIRAL2-GANIL. This paper is focused on the long beam transfer lines which will connect the different production sites to the DESIR experimental hall. Low energy beams will be delivered by the Low Energy Branch of the new Super Separator Spectrometer (S3), the SPIRAL2 production building and the upgraded SPIRAL1 facility. Special care must be taken about focalization devices, deflectors, kicker benders and orbit correctors in combination with beam diagnostic measurements in order to minimize the overall cost of the beam lines and to optimize their tuning. This process has started in 2013 and will continue in 2014. A prototype quadrupole structure is now under implementation. It will offer the possibility to validate the machining choices and to have feed-backs with the beam dynamic calculations. We also plan to make similar realisations for an electrostatic bender that will be tested at IPN Orsay.

\section{ACKNOWLEGMENTS}

This work is supported by the French research agency (ANR), through the program "Investissements d'Avenir", EQUIPEX Contract number ANR-11-EQPX-0012.

\section{References}

1. M.-G. Saint Laurent et al., SPIRAL PHASE-II, European RTT, Final report, Contract number ERBFMGECT980100, http://pro.ganil-spiral2.eu/spiral2, September (2001)

2. E. Petit, on behalf of the SPIRAL2 collaboration, Progress of the SPIRAL2 project, in proceedings of the IPAC conference, San Sebastian, Spain (2011), http://www.jacow.org/

3. B. Blank, The DESIR facility at SPIRAL2, Pramana - J. Phys 75 (2010) 343

4. B. Blank for the DESIR collaboration, The DESIR Facility, Letter of Intent for SPIRAL2 (2006), GANIL Caen-France, http://www.cenbg.in2p3.fr/desir/

5. F. Azaiez, S. Essabaa, F. Ibrahim and D. Verney, The ALTO facility at Orsay, NPN, 23, Issue 2 (2013)

6. The ISOLDE Facility, A Tool for Understanding the Strong Interaction, NPN, 20, Issue 4, (2010), http://www.scholarpedia.org/article/The ISOLDE facility

7. M. Trinczek, Beam Delivery And Future Initiatives at The ISAC Radioactive Ion Beam Facility, in proceedings of the PAC conference, Vancouver, BC, Canada, (2009), http://www.jacow.org/

8. H. Wollnik, Optics of Charged Particles, (Academic Press, INC. 1987)

9. F. Hinterberger, Ion Optics with Electrostatic Lenses, (CAS on Small Accelerators, CERN-2006012, 2006)

10. TraceWin code: http://irfu.cea.fr/Sacm/logiciels/index.php

11. R. Duperrier and D. Uriot, Application of the extreme value theory to beam loss estimates in the SPIRAL2 linac based on large scale Monte Carlo computations, Phys. Rev. Spe. Topics - Acce and Beams 9, 044202 (2006)

12. A. Septier and J. Van Acker, Les Lentilles Quadrupolaires Electrostatique, NIM 13335 (1961)

13. R. Baartman and D. Kaltchev, Short Quadrupole Parametrization, in proceeding of the PAC conference, Albuquerque, New Mexico, USA, (2007)

14. Opera-3d - electromagnetic design in three dimensions, http://www.cobham.com/aboutcobham/aerospace-and-security/about-us/antenna-systems/kidlington/products/opera-3d.aspx 